\title{
É possível encontrar inovação gerencial e práticas de gestão de pessoas voltadas à inovação em universidades federais?
}

Adauto de Vasconcelos Montenegro ${ }^{1}$

ANA PAUla MORENO PINHO ${ }^{2}$

ANTONIO CAUBi RIBEIRO TUPINAMBÁ 1

RAQUEL LIBÓRIO FEITOSA ${ }^{1}$

\author{
${ }^{1}$ Universidade Federal do Ceará (UfC) / Programa de Pós-Graduação em Psicologia, Fortaleza - CE, Brasil \\ ${ }^{2}$ Universidade Federal do Ceará (UFC) / Programa de Pós-Graduação em Administração e ControladoriA, Fortaleza - CE, Brasil
}

\section{Resumo}

Este artigo objetiva investigar a inovação gerencial e as práticas de gestão de pessoas voltadas à inovação em 2 universidades federais. Trata-se de uma pesquisa quantitativa, descritiva e de campo. Os principais resultados apontam que as práticas de gestão de pessoas voltadas à inovação com mais destaque foram "delegação" e "treinamento". Ademais, 55,7\% dos respondentes perceberam a inovação gerencial no contexto pesquisado, notadamente na área de gestão de pessoas. Evidenciaram-se contribuições para o conhecimento científico, ao investigar a inovação gerencial e de práticas voltadas à inovação no setor público, tema ainda pouco explorado na literatura; para a gestão de pessoas, ao investigar práticas da área; e para a prática de gestores, ao investigar tais fenômenos em áreas e categorias funcionais distintas.

Palavras-chave: Inovação gerencial. Práticas de gestão de pessoas voltadas à inovação. Setor público.

\section{Is it possible to find managerial innovation and people management practices focused on innovation in federal} universities?

\begin{abstract}
This article aimed to investigate managerial innovation and people management practices focused on innovation at two federal universities. The study was quantitative and descriptive and included field research. The main results showed that the people management practices focused on innovation with more prominence were delegation and training. In addition, 55.7\% of respondents perceived managerial innovation in the researched context, notably in the area of people management. Contributions to scientific knowledge were evidenced, in the investigation of managerial innovation and practices aimed at innovation in the public sector, a theme that is still little explored in the literature; for people management, when investigating practices in the area; and for the practice of managers, when investigating such phenomena in different areas and functional categories.
\end{abstract}

Keywords: Managerial innovation. People management practices focused on innovation. Public sector.

¿Es posible encontrar prácticas de innovación gerencial y gestión de personas enfocadas a la innovación en las universidades federales?

\section{Resumen}

Este artículo tuvo como objetivo investigar la innovación gerencial y las prácticas de gestión de personas centradas en la innovación en dos universidades federales. Fue una investigación cuantitativa, descriptiva y de campo. Los principales resultados mostraron que las prácticas de gestión de personas enfocadas a la innovación con mayor protagonismo fueron delegación y formación. Además, el 55,7\% de los encuestados percibió la innovación gerencial en el contexto investigado, especialmente en el área de gestión de personas. Los aportes al conocimiento científico se evidenciaron en la investigación de la innovación y prácticas gerenciales orientadas a la innovación en el sector público, tema que aún está poco explorado en la literatura; para la gestión de personas, al investigar prácticas del área; y para la práctica de los gestores, al investigar tales fenómenos en diferentes áreas y categorías funcionales.

Palabras clave: Innovación gerencial. Prácticas de gestión de personas enfocadas a la innovación. Sector público. 


\section{INTRODUÇÃO}

Questões ligadas às práticas de gestão estão presentes no cenário organizacional, relacionando-se com a busca da competitividade, da eficiência, da eficácia e da efetividade de ações, práticas e processos nas organizações. Tais práticas podem ser compreendidas como um conjunto de características e ações executadas no contexto da organização e que se associam a diversos aspectos, como gestão de pessoas (GP), estilos de liderança e estrutura organizacional. A inovação gerencial (IG), igualmente relevante nesse cenário, envolve tecnologias de caráter social no contexto organizacional e se conecta com dimensões, como atividades e práticas gerenciais, processos de gestão e estruturas organizacionais (Lopes, 2017).

Quando se analisa a literatura nesse campo, percebem-se poucos estudos tratando da IG e de práticas de GP voltadas à inovação no cenário brasileiro, sobretudo em organizações públicas (Pinho, M. R. R. Silva \& Evangelista, 2020; M. R. R. Silva, 2019). Nota-se também que muitos dos estudos privilegiam a visão do gestor sobre tais processos, em detrimento de demais trabalhadores que não ocupam posições de gestão.

Outra lacuna observada é a carência de sistematização de modelos de investigação de tais construtos em organizações públicas e privadas, de diferentes segmentos e configurações. Iniciativas importantes no cenário brasileiro são identificadas, por exemplo, por Janissek, Aguiar, Mello, Ferreira e Campos (2017), ao analisarem práticas inovadoras de gestão, e por Lopes (2017), ao investigar a IG e práticas de GP voltadas à inovação.

As práticas de GP envolvem recrutamento, seleção, treinamento, gestão do desempenho e recompensas, tendo como desafio ocupar um papel estratégico nas organizações, o que implica o envolvimento da área em processos de mudança e inovação (Armstrong, 2014; Dessler, 2002; Silvestre \& Araújo, 2013).

Becker e Huselid (2006) apontam que a IG ocorre num contexto organizacional com determinada complexidade e que diz respeito tanto à estratégia organizacional quanto a práticas de GP. Essas práticas, segundo os autores, podem também favorecer inovações gerenciais.

No contexto das Instituições de Ensino Superior (IES) públicas, as práticas de GP voltadas à inovação e a IG encontram desafios em sua plena implementação graças a questões culturais - tradicionalismo, excesso de burocracia, paternalismo e hierarquização - e normativas - regulamentações próprias do órgão - do setor (Ribeiro, 2017).

No campo das IES, observam-se estudos que tratam de determinadas práticas de gestão e de GP voltadas à inovação, mas sem uma sistematização tão clara e, muitas vezes, sem a discussão aprofundada do componente da inovação em tais contextos (Burigo \& Tosta, 2017; Genari, C. V. D. Ibrahim \& G. F. Ibrahim, 2017; Leal, A. C. F. Silva \& Dalmau, 2017; Reis, Freitas, Martins \& Oliveira, 2015; Tomazzoni, Costa, Santos, Espich \& Posser, 2017).

No campo da GP no setor público, também é evidenciada a necessidade de investir em mais pesquisas relacionadas com as práticas da área, aprimorar medidas de investigação de tais práticas (Demo, 2012; Demo, Fogaça \& Costa, 2018) e implementar um modelo de gestão estratégica de pessoas no setor público, dadas as suas especificidades (Bergue, 2020; Carmo, Assis, Martins, Saldanha \& Gomes, 2018).

Ainda considerando o contexto das IES públicas, há na literatura a necessidade de investigar e analisar se as práticas de gestão adotadas em tais contextos são adequadas e atendem aos objetivos organizacionais. Características como excesso de normalização, rigidez funcional, dificuldade de ser afetada por pressões externas e resistência a mudança podem limitar aspectos inovativos em práticas seguidas em tais ambientes (Ribeiro, 2017).

Considerando tais discussões e lacunas presentes na literatura, esta pesquisa objetiva investigar a IG e as práticas de GP voltadas à inovação em 2 universidades federais. Considera ainda 3 categorias ocupacionais atuantes nas IES públicas: servidores técnicos, gestores e terceirizados. 


\section{INOVAÇÃo GERENCIAL E PRÁTICAS DE GESTÃo DE PESSOAS VOLTADAS À INOVAÇÃo}

No campo da inovação, percebe-se uma multiplicidade de conceitos e variedade de abordagens para o estudo do fenômeno. O Manual de Oslo define inovação como um produto novo ou um processo melhorado (ou uma combinação) que apresenta diferença significativa em relação a produtos e processos anteriores, bem como que tal produto ou processo tenha sido disponibilizado para usuários potenciais ou postos em uso (Organisation for Economic Co-operation and Development [OECD], 2018).

Evidenciam-se estudos sobre inovação em diversos tipos e segmentos de organizações, podendo ocorrer em qualquer ambiente, porém as possibilidades de desenvolvimento estão sujeitas a diversas variáveis do contexto organizacional (Fernandes et al., 2016).

Queiroz, Albuquerque e Malik (2013) e Rodrigues e Veloso (2013) destacam que parece haver uma grande relação entre GP e inovação. Queiroz et al. (2013) apresentam um conjunto de características presentes em "empresas inovadoras" que remetem a processos relacionados à gestão, como descentralização, redução no número de níveis hierárquicos, redução no papel do staff corporativo, sistemas de comunicação interna e uso de grupos ad hoc. Pinho e Janissek (2019), mais recentemente, apontam alguns resultados de pesquisa acerca de cognições gerenciais, destacando um esquema de uma organização inovadora construído por gestores inseridos num contexto considerado muito inovador e atestando características como pensamento estratégico, ênfase nas pessoas, processos internos e liderança.

Além de tais discussões, é fundamental considerar que a inovação pode ser pensada, principalmente, com base no que é novo para determinado ambiente, organização ou contexto. Assim, uma ação pode ser pioneira num ambiente e já estar plenamente consolidada num contexto distinto (Queiroz et al., 2013; Spink, 2003).

Quando se discute especificamente a IG, é possível evidenciar a heterogeneidade e a imprecisão conceitual. Ven, Angle e Poole (2000) destacam que o processo de inovação, numa perspectiva gerencial, consiste na coordenação e no engajamento de pessoas para o desenvolvimento de novas ideias, com o objetivo de alcançar determinados resultados. O conceito de IG não deve estar limitado, portanto, à inovação tecnológica, e sim relacionado a processos e atividades que tenham a ver com organização e gestão. Apesar dessa diferenciação, é possível haver associação entre inovações do tipo tecnológico e gerencial com importantes consequências sobre o desempenho organizacional (Damanpour, 2014).

Outros estudos vão em direção semelhante, a exemplo do que apontam Birkinshaw, Hamel e Mol (2008), ao definirem IG como a "implementação de uma prática, processo, estrutura ou técnica de gestão que seja nova no estado da arte e que se destina a promover os objetivos organizacionais". Volberda, Bosch e Mihalache (2014) apontam uma sistematização de características distintivas da inovação em gestão, indicando seu potencial de aumento de desempenho e competitividade organizacional. Cerne, Kase e Skerlavaj (2016), ao investigarem a literatura sobre inovações não tecnológicas, apresentam como uma das conclusões a necessidade de aprofundar o estudo sobre inovação em gestão.

Mais recentemtente, Lopes sinaliza que o referido conceito pode ser entendido como "a introdução de algo novo para a firma nas seguintes dimensões: atividades e práticas gerenciais, processos de gestão e estruturas organizacionais" (Lopes, 2017, p. 29). As atividades e as práticas gerenciais correspondem à natureza das atividades e à forma pela qual são realizadas, em especial, pelos gestores de uma organização. Os processos de gestão, por sua vez, dizem respeito a rotinas, processos e procedimentos ligados às atividades desenvolvidas pelos gestores, como planejamento estratégico, comunicação interna e gestão do conhecimento. As estruturas organizacionais podem ser entendidas como a organização e a distribuição de níveis hierárquicos, linhas de comando e responsabilização.

De forma semelhante aos constutos "inovação" e "inovação gerencial", não existe consenso acerca da definição de "práticas de GP voltadas à inovação", sendo entendidas como ações apoiadas em modelos de gestão, relacionando-se com diversos aspectos organizacionais, como planejamento estratégico e tomada de decisão, e tendo o potencial de favorecer processos de inovação no contexto organizacional (Becker \& Huselid, 2006). Apesar dessa imprecisão, são identificadas iniciativas importantes na literatura, a exemplo daquelas propostas por Janissek et al. (2017) e Lopes (2017) no cenário brasileiro.

Janissek et al. (2017) propõem determinadas práticas inovadoras de gestão e as dividem em 3 domínios: gestão de pessoas, gestão organizacional e modernização de processos. Lopes (2017), por sua vez, com base em Laursen e Foss (2014), aponta 5 grupos de práticas de GP voltadas à inovação e citadas a seguir. "Delegação" pode ser entendida com base em aspectos como promoção de autonomia e descentralização de decisões. "Recrutamento e retenção" apontam aspectos relacionados 
tanto a recrutamento e seleção quanto a mecanismos de retenção de trabalhadores. "Incentivos" se referem a recompensas, de caráter individual e coletivo, associadas ao desempenho. "Treinamento" se refere aos diversos tipos e formatos de treinamento e capacitação, formais, internos e externos. "Comunicação", por fim, diz respeito aos fluxos de informação em diversos níveis e direções, assim como ao compartilhamento de informações. O modelo abordado por Lopes (2017, 2020), além de considerar as práticas de GP à inovação, aborda a adoção da IG.

No contexto do setor público, a IG assume contornos específicos. Castro, Isidro-filho, Menelau e Fernandes (2017) apontam facilitadores na inovação em organizações púbicas, como trabalho em equipe, desenvolvimento de pessoas e competências, assim como barreiras - por exemplo, resistênca, conflito de interesses e limitações de recursos humanos. Isidro (2018) apresenta o Modelo Integrado de Gestão Pública Inovadora para o Brasil (Gespublin), um modelo multinível com 4 dimensões: ambiente da inovação - variáveis contextuais ou organizacionais -, capacidades da inovação - competências organizacionais para mobilização de recursos em prol da inovação -, atividades de inovação - ciclo do processo de inovação - e resultados da inovação - consequências da inovação. Já Montezano e Isidro-Filho (2020) apresentam uma proposta de modelo multinível de competências para a gestão pública inovadora, apontando competências do setor público, organizacionais, de equipes e dos indivíduos, retomando o modelo de Isidro (2018).

O estado da inovação no setor público brasileiro apresenta características como produção científica limitada e pouco explorada, além de apropriação de práticas do mercado (Brandão \& Bruno-Faria, 2013). A inovação nas organizações públicas também está relacionada com a própria evolução e transformação das práticas de gestão desse campo. Apesar de todos os desafios envolvidos, são identificados aspectos vantajosos para a organização pública que adota práticas inovadoras como redução de custos, mais agilidade na realização de atividades e maior satisfação dos usuários-alvo dos serviços (Matos \& Nunes, 2016).

Considerando esse cenário, houve, em 1996, o lançamento do Concurso de Inovação na Gestão Pública Federal, pela Escola Nacional de Administração Pública (Enap), o que representou uma iniciativa importante de mapeamento de práticas, de caráter inovador, em diversas organizações. Tais práticas foram analisadas por Janissek et al. (2017), que as categorizou em gestão organizacional, gestão de pessoas e gestão de processos, identificando práticas como gestão por competências, avaliação de desempenho, aprendizagem contínua, informatização de sistemas, redesenho de processos e planejamento estratégico.

Dentro do grande espectro de organizações públicas, há as IES, que ocupam papel relevante na sociedade e sofrem influência de fatores de natureza político-institucional, econômica e cultural, além de apresentarem características de gestão como compulsão burocrática, excesso de normalização, corporativismo, rigidez funcional, dificuldade de ser afetada por pressões externas e resistência a mudança. Tais aspectos, muitas vezes, limitam o potencial de inovação nessas instituições, favorecendo a preservação de características tradicionais relacionadas ao modelo burocrático de gestão. Ribeiro (2017) menciona que universidades e demais IES se distanciam da estrutura e da dinâmica de uma empresa nos moldes tradicionais, assim como que seus objetivos nem sempre conseguem ser traduzidos e/ou delimitados com precisão. Além disso, a autora também aponta um desafio constante enfrentado sobretudo pelas universidades públicas: o contínuo impasse entre "adotar o novo e conservar o velho" (Ribeiro, 2017, p. 365). Tais instituições têm certo nível de complexidade, muitas vezes com características híbridas em modelos de gestão, combinando aspectos tradicionais e outros de caráter mais moderno (Seeber et al., 2014; Vieira, Bellen \& Fialho, 2006). Observa-se ainda uma complexidade na atuação por parte de gestores nas IES, o que exige diferentes competências, de caráter cognitivo, funcional, comportamental e político (Pereira \& A. L. C. Silva, 2011).

No que concerne às instituições públicas de ensino superior, tanto a IG quanto as práticas de GP voltadas à inovação apresentam determinadas características que se relacionam ao cenário de evolução de ações no setor público. Quanto à IG em IES públicas, há nuances específicas de sua implementação nesses locais, pois se associam questões relativas à gestão e ao papel da IES frente à atividade educacional, assim como há necessidade de aprimoramento no desenvolvimento de projetos de IG (Kalimullin, Youngblood \& Khodyreva, 2016).

Alguns estudos no âmbito internacional demonstram relações da IG em IES públicas com gestão do conhecimento, aprendizagem organizacional e práticas de gestão da qualidade (Aminbeidokhtia, Jamshidia \& Hoseinib, 2016; Ngoc-Tan \& Gregar, 2018; Sciarelli, Gheith \& Tani, 2020).

Quando se analisa a literatura acerca de práticas de GP em instituições públicas de ensino superior, evidenciam-se alguns estudos que não abordam essas práticas sob uma perspectiva voltada à inovação, o que indica lacunas na área. Tais pesquisas, no cenário nacional, abordam práticas de GP tradicionais, assim como outras sinalizam as lacunas citadas (Burigo \& Tosta, 2017; Genari et al., 2017; Leal et al., 2017; Montezano, N. B. Silva, Marques \& Isidro-Filho, 2019; Reis et al., 2015; Tomazzoni et al., 2017). 
Outros estudos também abordam práticas de GP com efeitos sobre diferentes construtos relevantes - comprometimento organizacional, produtividade, bem-estar e gestão do conhecimento - em instituições públicas de ensino superior em diversos países (Aboramadan, Albashiti, Alharazin \& Dahleez, 2020; Franco-Santos \& Doherty, 2017; Govender, Perumal \& Perumal, 2018; Nuryanto \& Pambukob, 2019; Szelagowska-Rudzka, 2018).

Apesar de conservar características tradicionais e burocráticas, percebem-se indícios na literatura de adoção de práticas de caráter gerencialista nas instituições públicas de ensino superior, o que é evidenciado por estudos que abordam diversas ações de GP em tais instituições (Burigo \& Tosta, 2017; Genari et al., 2017; Leal et al., 2017; Reis et al., 2015; Tomazzoni et al., 2017).

Entre as práticas de GP voltadas à inovação abordadas (Lopes, 2017), as de delegação, incentivos e treinamentos têm maior destaque no contexto público, enquanto as de comunicação e incentivos talvez tenham menor destaque, considerando aspectos engessantes relativos à legislação - no caso do recrutamento e da retenção - e aspectos organizacionais tradicionais nos fluxos de comunicação - no caso da comunicação. Com base em tais reflexões, formulou-se a seguinte hipótese: Os trabalhadores de diferentes categorias ocupacionais percebem a inovação gerencial, principalmente na área de gestão de oessoas, e as práticas de maior destaque são as de delegação, incentivos e treinamento.

\section{MÉTODO}

Trata-se de uma pesquisa quantitativa, descritiva e de campo. A área de análise é constituída por 2 universidades federais localizadas em 2 estados do Nordeste, escolhidas pelos seguintes critérios: acessibilidade geográfica da equipe de pesquisa e atuação de servidores e de terceirizados no corpo funcional administrativo.

Quanto ao perfil institucional, a primeira universidade, com número mais expressivo de respondentes, foi criada na década de 1950, tem atuação em toda a região territorial do estado em que está localizada e conta com 7 campi. A segunda foi criada na década de 1960, também tem atuação em todo o território do estado em que está localizada e tem 9 campi. As duas têm estrutura organizacional semelhante em termos de pró-reitorias e demais unidades administrativas.

Quanto à amostra, foram 371 respondentes de uma das universidades e 99 de outra, totalizando 470, divididos em 3 categorias ocupacionais: servidores técnicos, gestores e terceirizados, atuantes em áreas administrativas. A amostra obtida representou cerca de $8,5 \%$ dos servidores (técnicos e gestores) e 34,6\% dos terceirizados das 2 universidades. A disparidade na representatividade foi esperada, considerando o número expressivo de servidores, em comparação com os terceirizados. Houve representação de 10 campi das 2 universidades. Tendo em vista o objetivo deste artigo, o número de participantes é relativamente suficiente para um levantamento inicial das informações. Considerando as análises realizadas, aquela com maior demanda em relação ao tamanho da amostra é a fatorial exploratória. Não há consenso na literatura acerca do tamanho amostral mínimo para a realização de um exame fatorial exploratório. De modo geral, sugere-se uma proporção de 10 respondentes por item da escala, a presença de 100 respondentes ou tamanhos amostrais relacionados às cargas fatoriais (Beavers et al., 2013; Damásio, 2012; Hair, Black, Babin, Anderson \& Tatham, 2009).

De toda forma, as análises aqui descritas vão ao encontro de tais indicações, considerando que há, na presente pesquisa, uma proporção maior de participantes por item e por fator. Ademais, reconhecendo a complexidade do campo estudado e as implicações para a obtenção de uma amostra representativa para essas instituições, acredita-se que o número total de respondentes seja suficiente para alcançar o objetivo.

Os terceirizados foram incluídos visando explorar a percepção de diferentes atores no mesmo cenário organizacional. Os cargos ocupados pelos respondentes e os setores de lotação seguiram uma distribuição heterogênea, tendo em sua composição diversos setores das universidades.

A pesquisa resguardou a confidencialidade dos respondentes, tendo sido submetida ao Comitê de Ética em Pesquisa da Universidade Federal do Ceará (CEP-UFC), que emitiu parecer favorável de número 24747119.4.0000.5954.

O instrumento de pesquisa foi composto de: questões para investigação de dados sociodemográficos e ocupacionais; escala de práticas de GP voltadas à inovação, proposta por Lopes (2017), com base em Laursen e Foss (2011); e questões relacionadas à IG, à adoção e à complexidade da adoção, baseadas na proposta de Lopes (2017), com adaptações. As escalas foram do tipo likert de 6 pontos, variando de "discordo totalmente" a "concordo totalmente". Foram realizadas adaptações em relação a 
cada categoria ocupacional investigada e às organizações públicas, como denominações de áreas em que se percebe a IG adequando-as para a realidade de uma IES pública - e exclusão de itens referentes à inovação de produto e processo, mais relacionados ao âmbito privado.

Assim, os terceirizados não responderam a questões relativas às práticas de recrutamento e retenção e incentivos tangíveis e intangíveis, em função de os aspectos abordados em tais práticas fazerem referência à empresa contratante, e não à universidade em que atuam.

O tratamento dos dados sociodemográficos e ocupacionais foi realizado por meio da análise descritiva e comparativa das informações estatísticas. Quanto à escala, utilizou-se o alfa de Cronbach. Já para o agrupamento dos itens da mesma escala, foi realizada análise fatorial exploratória, observando-se valores da medida de Kaiser-Meyer-Olkin (KMO), do teste de esfericidade de Bartlett e da variância explicada pelo fator. Por fim, para a análise da percepção dos construtos, foram feitos ensaios descritivos e comparativos dos resultados estatísticos obtidos, além do exame de similitude, com a utilização do software Iramuteq.

\section{RESULTADOS}

Esta seção está dividida em: perfil da amostra, confiabilidade dos dados, agrupamento dos itens por meio da análise fatorial exploratória da escala de práticas de GP voltadas à inovação e resultados referentes à percepção das práticas de GP voltadas à inovação e da IG.

\section{Perfil da Amostra}

No que concerne à amostra, contou-se com a participação de 470 trabalhadores de 2 universidades públicas federais, com média de idade de 39 anos, a maior parte do gênero feminino $(58,03 \%)$, solteira $(44,30 \%)$ ou casada $(41,50 \%)$, com filhos (52,60\%), com 2 dependentes em média, com renda entre 2 mil e 5 mil reais (34\%) ou entre 5 mil e 8 mil reais $(30,60 \%)$. A maioria atua em regime de 40 horas semanais (70,20\%), assim como a média de tempo de serviço é de 9 anos e 6 meses na organização. Dos 470 respondentes, foram 301 servidores técnicos, 65 gestores e 104 terceirizados.

\section{Confiabilidade dos Dados}

Com o objetivo de verificar a homogeneidade do instrumento, foi realizada uma correlação item-total corrigida. A amplitude dos valores da correlação varia de 0,45 a 0,70 , satisfazendo ao ponto de corte estabelecido pela literatura ([ri.t $<0,30]$ ) (Pasquali, 2009). Diante desses resultados, foi possível concluir que os itens da escala de práticas de GP voltadas à inovação são homogêneos, ou seja, versam sobre o mesmo construto. Ademais, foram verificados os resultados relativos aos valores de alfa de Cronbach, que podem ser vistos na Tabela 1, sem a inclusão da prática "comunicação", em função dos resultados advindos da análise fatorial, descrita na próxima seção.

Tabela 1

Confiabilidade das dimensões abordadas

\begin{tabular}{c|c|c}
\hline Construto & Dimensões & Alfa de Cronbach \\
\hline \multirow{2}{*}{$\begin{array}{c}\text { Práticas de } \\
\text { GP voltadas } \\
\text { à inovação }\end{array}$} & Delegação & 0,87 \\
\cline { 2 - 3 } & Recrutamento e retenção & 0,78 \\
\cline { 2 - 3 } & $\begin{array}{c}\text { Incentivos tangíveis } \\
\text { e intangíveis }\end{array}$ & 0,79 \\
\cline { 2 - 3 } & Treinamento & 0,88 \\
\hline
\end{tabular}

Fonte: Elaborada com base em dados da pesquisa.

Verificou-se também a adequabilidade da matriz de dados para realização de uma análise fatorial, a qual foi considerada adequada $\left(\left[\chi^{2}(190)=3.883,05 ; p<0,001 ; K M O=0,91\right]\right)$ (Pasquali, 2009). 
É possível encontrar inovação gerencial e práticas de gestão de pessoas voltadas à inovação em universidades federais?
Adauto de Vasconcelos Montenegro | Ana Paula Moreno Pinho Antonio Caubi Ribeiro Tupinambá | Raquel Libório Feitosa

\section{Agrupamento dos Itens por Meio da Análise Fatorial Exploratória}

Frente aos resultados anteriores, deu-se sequência às análises. Com o objetivo de conhecer a estrutura fatorial da escala, foi feito um estudo fatorial exploratório, empregando-se o método de extração por componentes principais, sem rotação e número fixo de fatores. Para estabelecer a quantidade de fatores, utilizaram-se os critérios Kaiser, Cattell (scree-plot) e Horn (análise paralela). De acordo com o primeiro, são identificados 4 fatores, corroborados pelo último, visto que o quinto valor desse critério $(1,18)$ é superior ao quinto do critério de Kaiser $(0,81)$.

Considerando os resultados anteriores, procedeu-se a uma análise fatorial exploratória, empregando método de componentes principais, com rotação oblíqua, fixando dessa vez o número de fatores em 4, como indicado pelos critérios antes descritos, levando em conta $|0,40|$ como carga fatorial mínima para o pertencimento a um fator. Na Tabela 2, podem ser vistos esses resultados.

Tabela 2

Fatores da escala de práticas de GP voltadas à inovação

\begin{tabular}{|c|c|c|c|c|c|}
\hline Dimensão & Itens & & \multicolumn{3}{|c|}{ Fatores } \\
\hline & & 1 & 2 & 3 & 4 \\
\hline \multirow{4}{*}{ Delegação } & 1. Na minha organização, os empregados estão autorizados a tomar decisões. & $-0,86$ & 0,02 & $-0,04$ & $-0,05$ \\
\hline & 2. Os empregados estão autorizados a sugerir melhorias em suas atividades. & $-0,79$ & 0,08 & $-0,15$ & 0,19 \\
\hline & 3. A opinião dos empregados é valorizada pela organização. & $-0,82$ & 0,09 & 0,00 & 0,07 \\
\hline & 4. Os empregados estão envolvidos em equipes com alto grau de autonomia. & $-0,88$ & 0,02 & 0,08 & $-0,13$ \\
\hline \multirow{4}{*}{$\begin{array}{l}\text { Recrutamento } \\
\text { e retenção }\end{array}$} & 1. As contratações são feitas de maneira criteriosa. & $-0,07$ & 0,80 & $-0,17$ & 0,11 \\
\hline & $\begin{array}{l}\text { 2. Os empregados são selecionados com base em conhecimentos e } \\
\text { habilidades necessários à execução das atividades. }\end{array}$ & 0,08 & 0,85 & 0,05 & 0,04 \\
\hline & 3. Os empregados têm trajetórias claras de carreira dentro da organização. & $-0,13$ & 0,70 & 0,09 & $-0,02$ \\
\hline & 4. A organização prioriza a promoção interna dos atuais empregados. & $-0,24$ & 0,41 & 0,34 & $-0,05$ \\
\hline \multirow{5}{*}{$\begin{array}{l}\text { Incentivos } \\
\text { tangíveis e } \\
\text { intangíveis }\end{array}$} & 1. Existe uma ligação clara entre desempenho e recompensa & $-0,27$ & 0,00 & 0,64 & 0,00 \\
\hline & $\begin{array}{l}\text { 2. A remuneração está associada ao compartilhamento de conhecimentos } \\
\text { e/ou à sugestão de melhorias. }\end{array}$ & 0,10 & $-0,01$ & 0,87 & 0,00 \\
\hline & $\begin{array}{l}\text { 3. A remuneração está associada à melhoria de habilidades e/ou à } \\
\text { atualização de conhecimentos. }\end{array}$ & 0,16 & 0,22 & 0,73 & $-0,04$ \\
\hline & 4 Existe intercâmbio de informações entre os empregados das diversas áreas. & $-0,38$ & $-0,04$ & 0,42 & 0,17 \\
\hline & $\begin{array}{l}\text { 5. Os empregados têm a possibilidade de atuar em outras áreas por meio } \\
\text { de job rotation (rodízio de funções). }\end{array}$ & $-0,18$ & $-0,14$ & 0,62 & 0,18 \\
\hline \multirow{4}{*}{ Treinamento } & 1. Os empregados participam de treinamentos formais. & $-0,04$ & $-0,08$ & $-0,05$ & 0,88 \\
\hline & 2. As políticas e os programas de treinamento são abrangentes. & $-0,04$ & 0,08 & $-0,01$ & 0,80 \\
\hline & 3. Há treinamento para novos contratados. & 0,09 & 0,03 & $-0,02$ & 0,88 \\
\hline & 4. Há treinamentos voltados à solução de problemas. & 0,01 & 0,16 & 0,08 & 0,73 \\
\hline
\end{tabular}

Método de componentes principais, com rotação oblíqua.

Fonte: Elaborada com base em dados da pesquisa.

Com base na análise fatorial, observa-se que o fator 1 contempla os itens da prática de "delegação", o fator 2 compreende itens da prática de "recrutamento e retenção" e o fator 4 contempla itens da prática de "treinamento". Quanto ao fator 3, o único com 5 itens, agrupou objetos de 2 fatores presentes na versão original da escala: incentivos e comunicação. Os itens oriundos do fator "incentivos" foram: existe uma ligação clara entre desempenho e recompensa, a remuneração está associada ao compartilhamento de conhecimentos e/ou à sugestão de melhorias e a remuneração está associada à melhoria de habilidades e/ou à atualização de conhecimentos. 
Já os itens oriundos do fator "comunicação" foram: existe intercâmbio de informações entre os empregados das diversas áreas e os empregados têm a possibilidade de atuar em outras áreas por meio de job rotation (rodízio de funções). Esses itens, embora originalmente se refiram a processos de comunicação, também envolvem a possibilidade de aquisição de competências, por meio do trabalho em equipe e de troca de posições no contexto de trabalho, configurando-se como recompensas/incentivos de caráter intangível. Considerando que o novo fator compreendeu aspectos ligados a recompensas de caráter tangível - no caso, remuneração - e intangível - no caso, possibilidade de job rotation e intercâmbio de conhecimentos com colegas de trabalho-, convencionou-se denominá-lo "incentivos tangíveis e intangíveis".

Apesar da divergência da estrutura original, a escala apresentou propriedades psicométricas aceitáveis para o contexto pesquisado. A exclusão do fator "comunicação" no contexto desta pesquisa pode ser explicada por alguns aspectos. O primeiro deles é que a escala original foi validada antes em contexto distinto, composto majoritariamente por organizações privadas. O segundo diz respeito ao contexto específico desta pesquisa, IES públicas, campo que conta com aspectos desafiadores e limitadores em processos de comunicação, como questões estruturais e hierárquicas que ainda se aproximam de aspectos burocráticos e formais, o que é corroborado por Tomazzoni et al. (2017), os quais, em estudo realizado em universidades federais, evidenciaram a necessidade de treinamento em comunicação para servidores em estágio probatório, e por Genari et al. (2017), que identificaram deficiências de comunicação no processo de socialização de novos servidores.

\section{Percepção das Práticas de Gestão de Pessoas Voltadas à Inovação e da Inovação Gerencial}

Após a investigação de aspectos psicométricos da escala utilizada, verificou-se o nível de percepção das práticas de GP voltadas à inovação e da IG. É possível observar na Tabela 3 os escores, que variam de 1 a 5, relativos à percepção de cada prática, considerando a amostra total e as 3 categorias ocupacionais.

Tabela 3

Práticas de GP voltadas à inovação

\begin{tabular}{l|c|c|c|c}
\cline { 2 - 4 } & $\begin{array}{c}\text { Amostra total } \\
(n=470)\end{array}$ & $\begin{array}{c}\text { Técnico } \\
(n=301)\end{array}$ & $\begin{array}{c}\text { Gestor } \\
(n=65)\end{array}$ & $\begin{array}{c}\text { Terceirizado } \\
(n=104)\end{array}$ \\
\hline \multicolumn{1}{c|}{ Dimensão } & Média (DP) & Média (DP) & Média (DP) & Média (DP) \\
\hline Delegação & $3,23(1,07)$ & $3,19(1,04)$ & $3,65(1,07)$ & $3,11(1,09)$ \\
\hline Incentivos tangíveis e intangíveis & $2,36(0,91)$ & $2,31(0,89)$ & $2,56(0,98)$ & - \\
\hline Treinamento & $3,22(1,13)$ & $3,17(1,06)$ & $3,55(1,06)$ & $3,16(1,34)$ \\
\hline Recrutamento e retenção & $3,14(1,03)$ & $3,07(1,00)$ & $3,44(1,09)$ & - \\
\hline
\end{tabular}

Fonte: Elaborada com base em dados da pesquisa.

Com os dados apresentados na Tabela 3, evidencia-se, para a amostra total da pesquisa, que a prática com maior destaque é a de delegação, enquanto a de menor destaque é a relativa aos incentivos tangíveis e intangíveis. Esse resultado se repetiu para servidores técnicos e gestores. Já para os trabalhadores terceirizados, que responderam apenas às questões relativas às práticas de delegação e treinamento, a de maior destaque foi esta última. Ademais, "treinamento" ocupou a segunda posição de destaque para servidores técnicos e gestores.

Em relação à percepção da IG, evidenciam-se na Tabela 4 os resultados relativos à amostra total e às categorias ocupacionais. 
Tabela 4

Adoção da IG: amostra total e categorias ocupacionais

\begin{tabular}{|c|c|c|c|c|c|}
\hline \multirow[b]{2}{*}{ Variável } & \multirow[b]{2}{*}{ Níveis } & \multirow{2}{*}{$\begin{array}{l}\text { Amostra total } \\
(n=470)\end{array}$} & \multicolumn{3}{|c|}{ Categoria } \\
\hline & & & $\begin{array}{l}\text { Técnico } \\
(n=301)\end{array}$ & $\begin{array}{l}\text { Gestor } \\
(n=65)\end{array}$ & $\begin{array}{c}\text { Terceirizado } \\
(n=104)\end{array}$ \\
\hline \multirow{2}{*}{$\begin{array}{l}\text { Houve IG em sua } \\
\text { organização nos } \\
\text { últimos } 3 \text { anos? }\end{array}$} & Sim. & 262 & 161 & 41 & 60 \\
\hline & Não. & 208 & 140 & 24 & 44 \\
\hline \multirow{4}{*}{$\begin{array}{l}\text { Em que nível a IG } \\
\text { foi adotada? }\end{array}$} & Em toda a instituição. & 131 & 88 & 14 & 29 \\
\hline & Pró-reitoria/Diretoria/Gerência. & 65 & 40 & 15 & 10 \\
\hline & Departamento/Setor. & 52 & 25 & 9 & 18 \\
\hline & Projeto/Equipe. & 14 & 8 & 3 & 3 \\
\hline \multirow{4}{*}{$\begin{array}{l}\text { Qual é seu envolvimento } \\
\text { com a IG? }\end{array}$} & Nenhum, e meu trabalho não foi afetado. & 43 & 14 & 1 & 28 \\
\hline & Nenhum, mas meu trabalho foi afetado. & 127 & 92 & 16 & 19 \\
\hline & Fui integrante da equipe que implementou. & 66 & 40 & 16 & 10 \\
\hline & Fui responsável pela implementação. & 26 & 15 & 8 & 3 \\
\hline \multirow{2}{*}{$\begin{array}{l}\text { Em termos gerais, a } \\
\text { adoção da IG tem sido } \\
\text { benéfica à organização? }\end{array}$} & Sim. & 247 & 154 & 41 & 52 \\
\hline & Não. & 15 & 7 & 0 & 8 \\
\hline
\end{tabular}

Fonte: Elaborada com base em dados da pesquisa.

Para tais questões da Tabela 4, as 3 categorias responderam igualmente: 55,7\% ( $n=262$ ) dos respondentes perceberam algum processo de IG nas universidades pesquisadas nos últimos 3 anos. Destes, metade $(n=131)$ percebeu a IG em toda a instituição, enquanto $48 \%(n=127)$ relataram não ter tido envolvimento com o processo, mas tiveram o trabalho afetado por sua implementação, demonstrando que ela não é percebida de forma isolada e esporádica, impactando no modo de fazer as atividades, mesmo entre aqueles que não a idealizaram ou participaram formalmente de uma equipe de implementação.

Ao serem indagados sobre o efeito percebido da IG para a organização, 94,2\% dos trabalhadores afirmaram que ele foi benéfico, o que demonstra sua importância, além de ser um possível indicador de que processos, atividades e métodos foram afetados de forma satisfatória, talvez em relação à eficácia, à eficiência e/ou à efetividade.

Considerando cada categoria ocupacional, evidenciou-se para os servidores técnicos o seguinte: $86,9 \%(n=161)$ perceberam alguma IG nos últimos 3 anos. Entre eles, $54,6 \%(n=88)$ perceberam em toda a instituição; $57,1 \%(n=92)$ relataram não ter tido envolvimento com a IG, mas tiveram o trabalho afetado por sua implementação; e 95,6\% ( $n=154)$ perceberam que a IG foi benéfica à organização.

Entre os terceirizados, 57,6\% ( $n=60)$ perceberam alguma IG nos últimos 3 anos. Entre eles, $48,3 \%(n=29)$ perceberam na instituição como um todo, $46,6 \%(n=28)$ afirmaram que não tiveram envolvimento com a IG e que o trabalho não foi afetado e $86,6 \%(n=52)$ reconheceram que a IG teve efeito benéfico para a universidade.

Para os gestores, evidenciou-se o seguinte: $63 \%(n=41)$ perceberam algum processo de IG nos últimos 3 anos. Entre eles, $36,5 \%(n=15)$ apontaram que o processo ocorreu apenas no nível de pró-reitoria/diretoria/gerência; $39 \%(n=16)$ apontaram que não tiveram envolvimento direto com a IG, mas tiveram o trabalho afetado; e $39 \%(n=16)$ reconheceram a responsabilidade pela implementação da IG. Quanto à percepção acerca da IG, $100 \%(n=41)$ dos gestores consideraram que foi benéfica à organização.

Por último, todos os respondentes que perceberam algum processo de IG na universidade em que atuam nos últimos 3 anos informaram a(s) área(s) da universidade em que percebiam o processo. Tratava-se de uma questão de múltipla escolha, e o respondente poderia escolher 1 ou mais das 7 opções disponíveis, cujos resultados podem ser vistos na Tabela 5. 
Tabela 5

Distribuição da frequência das áreas em que os respondentes perceberam a IG: amostra total e categorias ocupacionais

\begin{tabular}{|c|c|c|c|c|c|c|c|c|}
\hline \multirow{3}{*}{ Áreas } & \multirow{2}{*}{\multicolumn{2}{|c|}{ Amostra total }} & \multicolumn{6}{|c|}{ Categoria } \\
\hline & & & \multicolumn{2}{|c|}{ Técnico } & \multicolumn{2}{|c|}{ Gestor } & \multicolumn{2}{|c|}{ Terceirizado } \\
\hline & $f$ & $\%$ & $f$ & $\%$ & $f$ & $\%$ & $\boldsymbol{F}$ & $\%$ \\
\hline Gestão de pessoas & 168 & 64,12 & 116 & 72,05 & 26 & 63,41 & 26 & 38,24 \\
\hline Planejamento, finanças/orçamento & 143 & 54,58 & 94 & 58,39 & 22 & 53,66 & 27 & 39,71 \\
\hline Assuntos estudantis & 92 & 35,11 & 60 & 37,27 & 18 & 43,90 & 14 & 20,59 \\
\hline Ensino & 89 & 33,96 & 58 & 36,02 & 17 & 41,46 & 14 & 20,59 \\
\hline Pesquisa e pós-graduação & 75 & 28,62 & 55 & 34,16 & 9 & 21,95 & 11 & 16,18 \\
\hline Extensão & 64 & 24,42 & 44 & 27,33 & 11 & 26,83 & 9 & 13,24 \\
\hline Outra & 37 & 14,12 & 25 & 15,53 & 3 & 7,32 & 9 & 13,24 \\
\hline
\end{tabular}

Fonte: Elaborada com base em dados da pesquisa.

Com a análise da Tabela 5, depreende-se que os servidores técnicos e os gestores percebem a IG, principalmente, na área de GP, seguida pela área de planejamento, finanças/orçamento e pela área de assuntos estudantis. As áreas de pesquisa e pós-graduação e extensão são as menos significativas. Por outro lado, os terceirizados percebem mais a IG na área de planejamento/finanças/orçamento, seguida pela de GP. Com o objetivo de avaliar a ocorrência simultânea das áreas em que se percebem a IG, realizou-se a análise de similitude. Os resultados podem ser observados na Figura1.

Figura 1

Análise de similitude das áreas de percepção da IG

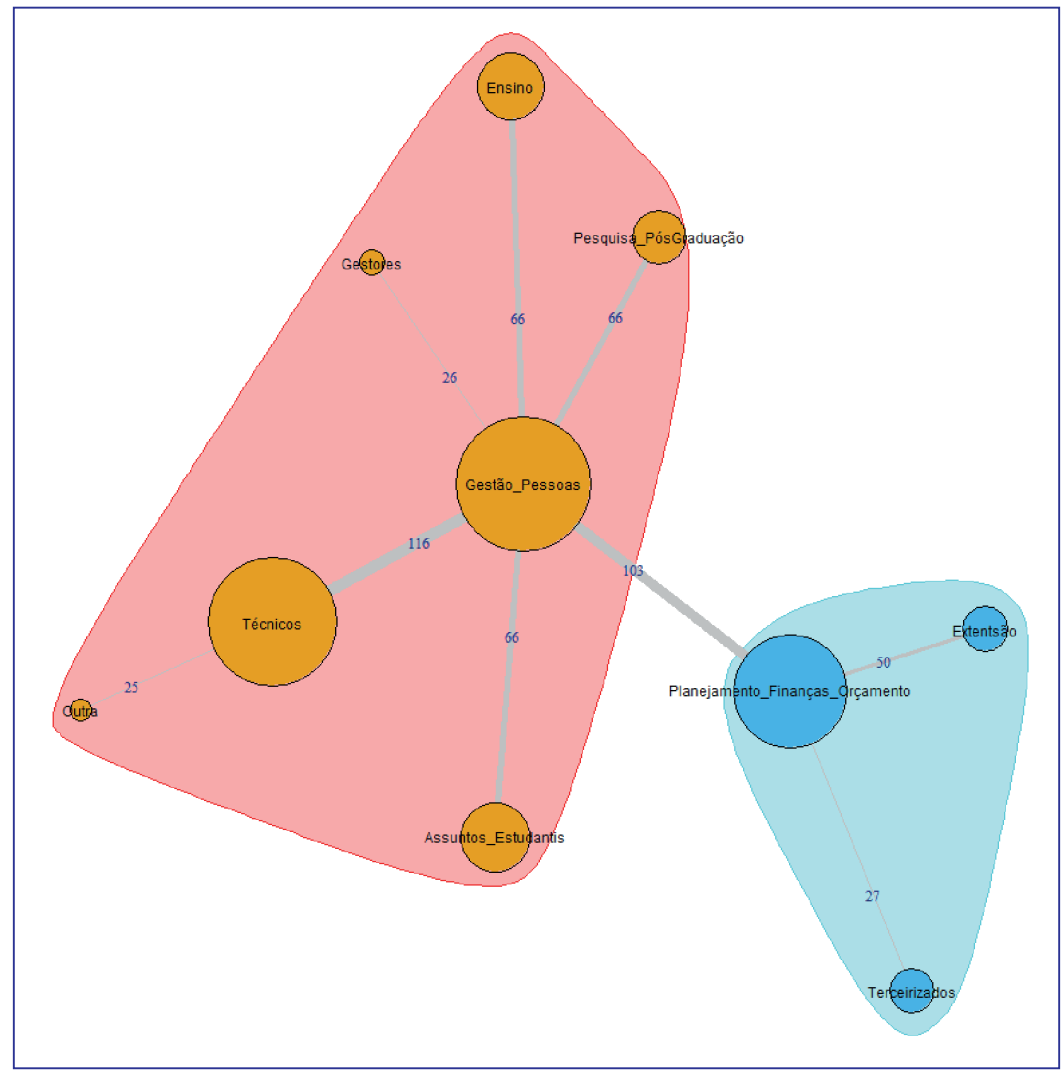

Fonte: Elaborada com base em dados da pesquisa. 
Destaca-se que as cores formam "comunidades". Cada uma dessas comunidades representa uma conexão frequente ou comum, enquanto os círculos são as áreas em que os respondentes poderiam assinalar. Quanto maior o diâmetro, mais frequente foi a percepção da inovação nessa área. Ademais, as linhas que conectam os círculos indicam quantas vezes esses 2 foram marcados por um respondente de forma seguida, observando também o número de ocorrências.

A Figura 1, portanto, apresenta 2 comunidades principais, sendo a primeira organizada ao redor da área de GP, à qual se ligam as áreas de pesquisa e pós-graduação, ensino, assuntos estudantis e outras áreas. À comunidade em questão estão ligadas ainda as categorias ocupacionais de técnicos e de gestores, concluindo que a IG é percebida principalmente por servidores técnicos e gestores na área de GP. A segunda comunidade é organizada ao redor de planejamento, finanças e orçamento, ligando-se à categoria de terceirizados, concluindo que estes percebem mais a IG na área de planejamento, finanças e orçamento, em especial.

\section{DISCUSSÃO}

Quanto às práticas mais percebidas por técnicos e gestores, tiveram ênfase a delegação e o treinamento. O destaque para a prática de delegação está em consonância com o estudo de Tierney et al. (2019), o qual demonstrou que práticas relacionadas à maior promoção de autonomia, descentralização, liberdade e participação estão ligadas à inovação ascendente (bottom-up innovation) no contexto do serviço público. Também está em consonância com achados de uma pesquisa cujos resultados apontaram que o envolvimento de equipes no contexto de trabalho universitário tem efeitos positivos sobre o bem-estar (Franco-Santos \& Doherty, 2017).

Já em relação à prática de treinamento, o resultado se relaciona com evidências destacadas por Nuryanto e Pambukob (2019), ao apontarem as ações de treinamento como centrais e relevantes em práticas de GP em universidades públicas. Govender et al. (2018) também destacaram a gestão do conhecimento como aliada importante das práticas de GP em universidades públicas de diferentes países.

A prática também percebida por técnicos e gestores, após as de delegação e treinamento, foi a de recrutamento e retenção, o que se relaciona com estudos que vêm se voltando para a análise de perfis de cargos no contexto das universidades, como os de Leal et al. (2017), que pesquisaram aspectos ligados à avaliação de competências de novos servidores em universidades públicas, para o cargo de secretário executivo, bem como questões relativas à retenção.

Os terceirizados perceberam mais a prática de treinamento do que a de delegação, o que pode ser explicado pelo fato de a maioria deles ter limitações em sua atuação, graças a questões legais, contratuais, e à empresa contratante. Tais aspectos podem limitar assuntos relativos à liberdade e à autonomia (Chambel, 2012).

A prática menos percebida pelas diferentes categorias ocupacionais, por sua vez, foi a de incentivos tangíveis e intangíveis. No que concerne aos primeiros, o resultado pode ser explicado pela dificuldade na implementação de regimes de remuneração baseados em competências, habilidades e/ou aspectos relacionados a resultados, em função de limitações da legislação relativa ao setor público, assim como a previsibilidade dos planos de carreira, em que as mudanças de remuneração estão associadas a períodos e requisitos específicos, e, por vezes, não relacionados a aspectos como competências e/ou resultados. Quanto aos incentivos intangíveis, muitas vezes oportunidades como job rotation ou equipes envolvidas em projetos são pouco presentes em algumas universidades. Destaca-se, porém, que, para a face de incentivos intangíveis, há maior margem de atuação para intervenções por parte da gestão (Montezano et al., 2019; Montezano \& Isidro-Filho, 2020; Pinto \& Behr, 2015).

Comparando tais resultados relativos à percepção das práticas de GP voltadas à inovação, àqueles encontrados na pesquisa de Lopes (2017), realizada numa amostra com 76,2\% de organizações privadas, observam-se semelhanças e diferenças. "Treinamento" também obteve destaque nos achados de Lopes (2017), obtendo a segunda maior média. Por outro lado, "recrutamento e retenção" obteve a maior média - enquanto na pesquisa atual obteve a terceira maior -, ao passo que "delegação" obteve a terceira maior - enquanto no estudo atual obteve a maior -, ou seja, ficaram em posições opostas nas 2 pesquisas. Esse resultado pode ser explicado pela maior incidência e dinamismo de processos de recrutamento nas organizações privadas, assim como maior flexibilidade nos processos de retenção atrelados à remuneração variável, o que ocorre de forma diferente no âmbito público, sujeito à legislação. 
Quanto à percepção de IG na pesquisa atual, mesmo diante de um cenário no qual estão presentes diversas características burocráticas, de resistência a mudança e excesso de formalismo, ainda foi possível verificar um alto índice de percepção da IG nas universidades pesquisadas (Ribeiro 2017). Esse resultado se relaciona a outros estudos no âmbito internacional. Numa pesquisa junto a 30 universidades públicas, os resultados evidenciaram que a utilização do conhecimento - dimensão da gestão do conhecimento - se relaciona à IG (Ngoc-Tan \& Gregar, 2018). Já o estudo de Aminbeidokhtia et al. (2016) demonstrou relação entre a aprendizagem organizacional e a IG também em universidades públicas. Tais achados demonstram que, apesar das limitações legais e culturais, as IES públicas podem vivenciar implementação de IG em seus contextos de trabalho em diferentes países.

Ao comparar a percepção da IG com os resultados encontrados por Lopes (2017), que teve amostra predominante no âmbito privado, houve uma percepção maior de IG na pesquisa realizada pelo autor $(64,2 \%)$ - cerca de 8 pontos percentuais a mais do que na atual pesquisa. Quanto à abrangência, os achados de Lopes (2017) demonstraram que 63,7\% das organizações adotaram IG, sendo que, na atual pesquisa, apenas $50 \%$ dos respondentes perceberam o alcance da IG em toda a instituição. Esse resultado pode ser explicado por características tradicionais e pelo modelo burocrático em universidades brasileiras. Lopes et al. (2018) apontam alguns fatores determinantes da IG: contingenciais (relacionados o tipo de organização, idade da organização), configuração estrutural (níveis de formalização, centralização, comunicação etc.) e interações externas e internas (interesses, estruturas de poder, conhecimentos sobre gestão etc.). Esses fatores, dentro do contexto de uma universidade federal, permeados por aspectos burocráticos e tradicionais, podem limitar o potencial inovativo, explicando a percepção de IG menor, quando comparada com a percepção na iniciativa privada nos achados de Lopes (2017).

Quanto à percepção menos significativa da IG por parte dos terceirizados, esta pode ser explicada pela forma de contrato estabelecido com a universidade, de caráter mais frágil, o que impossibilita a participação plena em todas as decisões. Acrescenta-se também a relação "dupla" estabelecida com a empresa contratante e com a organização em que atua (Chambel, 2012), o que pode limitar o acesso a determinadas práticas de gestão. Isso quer dizer que nem sempre as práticas de GP da empresa em que atuam são aplicadas a eles, pelo menos não da mesma forma e alcance visto para os servidores públicos.

O destaque conferido à área de GP, quando se perguntou a área em que mais se percebe a IG, está em consonância com estudos anteriores que evidenciam a presença massiva de diferentes práticas de GP no contexto das instituições públicas de ensino superior (Burigo \& Tosta, 2017; Moura \& Souza, 2016; Tomazzoni et al., 2017). Esse resultado, guardadas as devidas diferenças entre as pesquisas, também se repetiu na pesquisa de Lopes (2017), em que foi evidenciado que os temas/as áreas de gestão que ganharam destaque foram "estratégia e recursos humanos", com 70,4\% e 54,3\% das inovações realizadas, respectivamente, reforçando a importância da área em relação à adoção de inovações gerenciais. Ademais, esse resultado se alinha aos achados que demonstram relações entre IG e a área de GP em organizações privadas (Pinho et al., 2020; Queiroz et al., 2013; M. R. R. Silva, 2019), demonstrando a ocorrência da relação entre os fenômenos em contextos distintos.

Por fim, é possível apontar que a hipótese de pesquisa - os trabalhadores de diferentes categorias ocupacionais percebem a inovação gerencial principalmente na área de gestão de pessoas, e as práticas de maior destaque são as de delegação, incentivos e treinamento - foi parcialmente confirmada, tendo em vista a significativa percepção de IG e o destaque encontrado para as práticas de delegação e treinamento.

\section{CONSIDERAÇÕES FINAIS}

Este estudo objetivou investigar a IG e as práticas de GP voltadas à inovação em 2 universidades federais. Diante do objetivo previsto, foi possível identificar as práticas de GP mais percebidas pela amostra pesquisada - delegação e treinamento -, assim como a menos percebida - incentivos tangíveis e intangíveis. Foi possível também evidenciar que uma parcela significativa do corpo funcional percebe o processo de IG, em particular, na área de GP das universidades pesquisadas.

Quanto às contribuições teóricas, destaca-se primeiro a descoberta de uma estrutura fatorial distinta relativa às práticas de GP voltadas à inovação investigadas nesta pesquisa, a qual possibilitou a emergência do fator "incentivos tangíveis e intangíveis" e excluiu o fator "comunicação", presente na versão original. Outra contribuição, no nível teórico, foi a possibilidade de investigar aspectos relacionados à adoção da IG, baseados na percepção de trabalhadores inseridos em 3 categorias ocupacionais distintas, verificando semelhanças e diferenças, sobretudo em questões específicas relativas aos terceirizados. Por fim, 
os resultados encontrados, ao serem comparados com estudos do âmbito internacional, puderam encontrar aproximações no que concerne à percepção da IG em IES públicas. Já na comparação com a iniciativa privada, os resultados puderam ser contextualizados com base em questões relacionadas com os desafios enfrentados pelas universidades no campo da gestão, como resistência a mudança, dificuldade de implementar o novo e forte aderência a aspectos tradicionais e burocráticos.

Outra contribuição teórica incidiu na análise do resultado que evidenciou a percepção maior da IG na área de GP, o que pode sinalizar a necessidade de discutir o campo da inovação, tendo como base contribuições da literatura da gestão estratégica de recursos humanos/gestão estratégica de pessoas, de forma mais efetiva.

No que concerne às contribuições para as IES pesquisadas, aponta-se primeiro que identificar a percepção mais frágil da prática de incentivos tangíveis e intangíveis pode motivar os gestores a delinear estratégias de recompensas ou de reconhecimento do desempenho de seu corpo funcional. Diante das limitações normativas do setor (Ribeiro, 2017), talvez o investimento maior deva ocorrer no âmbito dos incentivos intangíveis, buscando, por exemplo, a implantação ou a consolidação da gestão por competências, que ainda se mostra com desafios relevantes no campo da IES públicas (Montezano et al., 2019; Montezano \& Isidro-Filho, 2020; Pinto \& Behr, 2015)

A percepção significativa de IG na área de GP se configura como uma pista importante para intervenções organizacionais. Esse resultado demonstra 2 aspectos importantes. Um deles é a potencialidade de considerar a GP como área em que se inova ou passível de "sofrer inovações", ou de "inovar-se" e "reinventar-se". Esse resultado tem consequências práticas importantes, pois a área de GP pode ser uma peça fundamental na disseminação de processos de inovação, assim como no apoio a processos de mapeamento, identificação e implementação de inovações gerenciais, tornando um setor de referência na temática dentro das universidades.

Já para o setor público, de forma geral, os achados deste estudo podem sugerir que a IG, possivelmente, se constitui como uma das principais manifestações da inovação em organizações públicas, mediante a superação de práticas e processos estritamente tradicionais e burocráticos e baseado em tentativas de melhoria e modernização de tais práticas e processos. Além de visualizar a óptica de práticas e processos, é fundamental considerar que a IG deve estar acompanhada de inovações no serviço público propriamente dito, ou seja, inovações na atividade-fim do serviço, destinada ao usuário do serviço público. Apesar de os resultados do estudo apontarem para indícios de implementação da IG nas organizações públicas pesquisadas, ainda é necessário inovar nos princípios e na filosofia de gestão no contexto do setor público.

Entre as limitações da pesquisa, estão a quantidade reduzida de organizações pesquisadas, assim como a não investigação de aspectos qualitativos relacionados com percepção de processos e ações ligadas à inovação. Ademais, destaca-se a dificuldade de acesso a terceirizados e gestores na coleta de dados, o que se refletiu na distribuição amostral entre as categorias ocupacionais. Sugere-se, para futuras pesquisas, a abordagem qualitativa de tais fenômenos no setor público, assim como em organizações públicas de outros segmentos, com a escala utilizada, visando realizar comparações entre organizações com características distintas, bem como aprofundar a capacidade da área de GP inovar.

\section{AGRADECIMENTOS}

Os autores agradecem ao Conselho Nacional de Desenvolvimento Científico e Tecnológico (CNPq) pelo suporte financeiro dado ao desenvolvimento da pesquisa. Projeto CNPq no 438071/2018-2. 
É possível encontrar inovação gerencial e práticas de gestão de pessoas voltadas à inovação em universidades federais?
Adauto de Vasconcelos Montenegro | Ana Paula Moreno Pinho Antonio Caubi Ribeiro Tupinambá| Raquel Libório Feitosa

\section{REFERÊNCIAS}

Aboramadan, M., Albashiti, B., Alharazin, H., \& Dahleez, K. A. (2020). Human resources management practices and organizational commitment in higher education: the mediating role of work engagement. International Journal of Educational Management, 4(1), 154-174.

Aminbeidokhtia, A., Jamshidia, L., \& Hoseinib, A. M. (2016). The effect of the total quality management on organizational innovation in higher education mediated by organizational learning. Studies in Higher Education, 41(7), 1153-1166.

Armstrong, M. (2014). A handbook of human resource management practice (13a ed.). London, UK: Kogan Page.

Beavers, A. S., Lounsbury, J. W., Richards, J. K., Huck, S. W., Skolits, G. J., \& Esquivel, S. L. (2013). Practical considerations for using exploratory factor analysis in educational research. Practical Assessment, Research, and Evaluation, 18(1), 1-13.

Becker, B. E., \& Huselid, M. A. (2006). Strategic human resources management: where do we go from here? Journal of Management, 32(6), 898-925.

Bergue, S. T. (2020). Gestão estratégica de pessoas no setor público. Belo Horizonte, MG: Fórum.

Birkinshaw, J., Hamel, G., \& Mol, M. J. (2008). Management innovation. Academy of Management Review, 33(4), 825-845.

Brandão, S., \& Bruno-Faria, M. (2013). Inovação no setor público: análise da produção científica em periódicos nacionais e internacionais da área de administração. Revista de Administração Pública, 47(1), 227-248.

Burigo, E. M., \& Tosta, K. (2017). Fatores que interferem no desempenho dos servidores ocupantes do cargo de administrador da UFSC durante o estágio probatório. Revista Práticas em Gestão Universitária, 1(2).

Carmo, L. J. O, Assis, L. B., Martins, M. G., Saldanha, C. C. T., \& Gomes, P. A. (2018). Gestão estratégica de pessoas no setor público: percepções de gestores e funcionários acerca de seus limites e possibilidades em uma autarquia federal. Revista do Serviço Público, 69(2), 163-191.

Castro, C. M. S., Isidro-filho, A., Menelau, S., \& Fernandes, A. S. A. (2017). Antecedentes de inovações em organizações públicas do poder executivo federal. Cadernos de Gestão Pública, 22, 126-141.

Cerne, M., Kase, R., \& Skerlavaj, M. (2016). Non-technological innovation research: evaluating the intellectual structure and prospects of an emerging field. Scandinavian Journal of Management, 32(2), 69-85.

Damásio, B. F. (2012). Uso da análise fatorial exploratória em psicologia: avaliação psicológica. Interamerican Journal of Psychological Assessment, 11(2), 213-228.

Demo, G. (2012). Políticas de gestão de pessoas nas organizações: estado da arte, produção nacional, agenda de pesquisa, medidas e estudos relacionais. São Paulo, SP: Atlas.

Demo, G., Fogaça, N., \& Costa, A. C. (2018). Políticas e práticas de gestão de pessoas nas organizações: cenário da produção nacional de primeira linha e agenda de pesquisa. Cadernos EBAPE.BR, 16(2), 250-263.
Dessler, G. (2002). Human resource management (9a ed.). New Jersey, NJ: Prentice Hall.

Damanpour, F. (2014). Footnotes to research on management innovation. Organization Studies, 35(9), 1265-1285.

Fernandes, F. M. B., Moreira, M. R., Ribeiro, J. M., Ouverney, A. M., Oliveira, F. J. F., \& Moro, M. F. A. (2016). Inovação em ouvidorias do SUS: reflexões e potencialidades. Ciência \& Saúde Coletiva, 21(8), 2547-2554.

Franco-Santos, M., \& Doherty, N. (2917). Performance management and well-being: a close look at the changing nature of the UK higher education workplace. The International Journal of Human Resource Management, 28(16), 1-32.

Genari, D., Ibrahim, C. V. D., \& Ibrahim, G. F. (2017). A percepção dos servidores públicos sobre a socialização organizacional: um estudo no Instituto Federal de Educação, Ciência e Tecnologia do Rio Grande do Sul. Holos, 33(5), 313-328.

Govender, L. N., Perumal, R., \& Perumal, S. (2018). Knowledge management as a strategic tool for human resource management at higher education institutions. South African Journal of Information Management, 20(1), 1-10.

Hair, J. F., Jr., Black, W. C., Babin, B. J., Anderson, R. E., \& Tatham, R. L. (2009). Análise multivariada de dados. Porto Alegre, RS: Bookman Editora.

Isidro, A. (2018). Gestão pública inovadora: um guia para a inovação no setor público. Curitiba, PR: Editora CRV.

Janissek, J., Aguiar, C. V. N., Mello, T. A. B., Ferreira, R. S., \& Campos, M. S. (2017). Práticas inovadoras de gestão no contexto das universidades públicas brasileiras: validação da escala para medir seu grau de importância e adoção. Revista do Serviço Público, 68(2), 259-284.

Kalimullin, A. M., Youngblood, V. T., \& Khodyreva E. A. (2016). The system of management of innovation projects at a higher education. International Journal of Environmental \& Science Education, 11(5), 613-622.

Laursen, K., \& Foss, N. J. (2014). Human resource management practices and innovation. In: Dodgson, M., \& Gann, D. Handbook of innovation management. Oxford, UK: Oxford University Press.

Leal, F. G., Silva, A. C. F., \& Dalmau, M. B. L. (2017). Recrutamento e seleção por competências para o cargo de secretário executivo. Revista Práticas em Gestão Universitária, 1(2), 1-23.

Lopes, D. P. T. (2017). Inovação gerencial na perspectiva da gestão de recursos humanos (Tese de Doutorado). Universidade Federal de Minas Gerais, Belo Horizonte, MG

Lopes, D. P. T. (2020). Human resource management perspective on innovation. In V. Škudiene, J. Li-Ying, \& F. Bernhard (Orgs.), Innovation management: perspectives from strategy, product, process and human resources research. Cheltenham, UK: Edward Elgar Publishing.

Lopes, D. P. T., Santos, S. R. M., Silva, V. H. R., \& Martins, G. S. (2018). Fatores determinantes da inovação gerencial: uma proposta para análise e investigação. Revista Eletrônica Gestão e Sociedade, 12, 2541-2563. 
É possível encontrar inovação gerencial e práticas de gestão de pessoas voltadas à inovação em universidades federais?
Adauto de Vasconcelos Montenegro | Ana Paula Moreno Pinho Antonio Caubi Ribeiro Tupinambá | Raquel Libório Feitosa
Matos, A. A., \& Nunes, A. M. (2016). Inovação da gestão dos hospitais públicos no sistema de saúde português. Revista de Gestão em Sistemas de Saúde, 5(2), 14-23.

Montezano, L., \& Isidro-Filho, A. (2020). Proposta de modelo multinível de competências para gestão pública inovadora. Future Studies Research Journal - Future, 12(2), 355-378.

Montezano, L., Silva, N. B., Marques, F. B., \& Isidro-Filho, A. (2019). Aspectos determinantes da implantação da gestão por competências em institutos federais. Revista Gestão Universitária da América Latina, 12, 21-44.

Moura, A. L. N., \& Souza, B. C. (2016). Gestão estratégica de pessoas na administração indireta do setor público federal: na prática, ainda um discurso. Revista do Serviço Público, 67(4), 575-602.

Ngoc-Tan, N., \& Gregar, A. (2018). Impacts of knowledge management on innovation in higher education institutions: an empirical evidence from Vietnam. Economics and Sociology, 11(3), 301-320.

Nuryanto, M., \& Pambukob, Z. B. (2019). A study on the effect of human resource empowerment on productivity: evidence from Indonesian higher education. Management Science Letters, 9, 1977-1986.

Organisation for Economic Co-operation and Development. (2018). Oslo Manual 2018: guidelines for collecting, reporting and using data on innovation. Recuperado de www.oecdilibrary.org/ docserver/9789264304604en.pdf?expires $=1604095233 \&$ id=id\& accname=guest $\&$ checksum=F2CEA1B7BFF46C4170D4074054228472

Pasquali, L. (2009). Instrumentação psicológica: fundamentos e práticas. Porto Alegre, RS: Artmed.

Pinho, A. P. M., \& Janissek, J. A. J. (2019). Cognição e ação: explicando as bases cognitivas do comportamento gerencial. In A. C. R. Tupinambá, \& A. G. Rodríguez (Orgs.), Liderança e empreendedorismo em perspectiva intercultural. Fortaleza, CE: Edições UFC.

Pinho, A. P. M., Silva, M. R. R., \& Evangelista, N. R. S. (2020). Cognições gerenciais sobre inovação gerencial e gestão de pessoas. Revista de Psicologia, 11(1), 49-58.

Pinto, J. F., \& Behr, R. R. (2015). Contradições na avaliação de desempenho dos servidores técnico-administrativos em educação na universidade pública. Cadernos EBAPE.BR, 13(4), 795-820.

Pereira, A. L. C., \& Silva, A. B. (2011). As competências gerenciais nas instituições federais de educação superior. Cadernos EBAPE.BR, 9(esp), 627-647.

Queiroz, A. C. S., Albuquerque, L. G., \& Malik, A. M. (2013). Gestão estratégica de pessoas e inovação: estudos de caso no contexto hospitalar. Revista de Administração, 48(4), 658-670.

Reis, C. Z. T., Freitas, A. M. R., Martins, S., \& Oliveira, A. R. (2015). Aspectos impactantes no dimensionamento da força de trabalho em uma instituição de ensino superior. Revista Gual - Gestão Universitária na América Latina, 8(2), 28-49.

Ribeiro, M. C. (2017). A natureza da gestão universitária: influência de aspectos político-institucionais, econômicos e culturais. Revista Internacional de Educação Superior - Riesup, 3(2), 357-378.

Rodrigues, A. F., \& Veloso, A. (2013). Contribuições da gestão de recursos humanos para a criatividade e a inovação organizacional. Revista Psicologia, Organizações e Trabalho, 13(3), 283-308.

Sciarelli, M., Gheith, M. H., \& Tani, M. (2020). The relationship between quality management practices, organizational innovation, and technical innovation in higher education. Quality Assurance in Education, 28(3), 137-150.

Seeber, M., Lepori, B., Montauti, M., Enders, J., Boer, H., Weyer, E. ... Reale, E. (2014). European universities as complete organizations? Understanding identity, hierarchy and rationality in public organizations. Public Management Review, 17(10), 1-31.

Silva, M. R. R. (2019). Inovar também é sobre pessoas: um estudo sobre práticas de gestão de pessoas e inovações gerenciais (Dissertação de Mestrado). Universidade Federal do Ceará, Fortaleza, CE.

Silvestre, H. C., \& Araújo, J. F. (2013). Coletânea em administração pública. Lisboa, Portugal: Escolar Editora.

Spink, P. (2003). Inovação na perspectiva dos inovadores: a experiência do Programa Gestão Pública e Cidadania. Cadernos EBAPE.BR, 1(2), 1-13.

Szelagowska-Rudzka, K. (2018). Human resources management in higher education institutions in Poland. Management, 22(1), 208-225.

Tierney, E., Hannigan, A., Kinneen, L., May, C., O’Sullivan, M., King, R. ..., \& Farlane, A. (2019). Interdisciplinary team working in the Irish primary healthcare system: analysis of "invisible" bottom up innovations using Normalisation Process Theory. Health Policy, 123(1), 1083-1092.

Tomazzoni, G. C., Costa, V. M. F., Santos, A. S., Espich, D., \& Posser, T. G. (2017). O processo de efetivação do servidor: um estudo acerca do estágio probatório nas universidades. Revista Gual - Gestão Universitária na América Latina, 10(1), 62-78.

Ven, A. H. V., Angle, H. L., \& Poole, M. S. (2000). Research on the management innovation: the Minnesota studies. Oxford, UK: Oxford University Press.

Vieira, E. M. F., Bellen, H. M., \& Fialho, F. A. P. (2006). Universidade em tempos de mudança. Cadernos EBAPE.BR, 4(3), 1-7.

Volberda, H. W., Bosch, F. A. J. V de, \& Mihalache, O. R. (2014). Advancing management innovation: synthesizing processes, levels of analysis, and change agents. Organization Studies, 35(9), 1245-1264. 
Adauto de Vasconcelos Montenegro

ORCID: https://orcid.org/0000-0002-6952-0739

Doutor em Psicologia pelo Programa de Pós-Graduação em Psicologia da Universidade Federal do Ceará (UFC); Psicólogo do Instituto de Educação, Ciência e Tecnologia do Ceará (IFCE).E-mail: adauto_montenegro@hotmail.com

Ana Paula Moreno Pinho ORCID: https://orcid.org/0000-0001-9671-8559

Doutora em Administração; Professora da Universidade Federal do Ceará no Programa de Pós-graduação em Administração e Controladoria (PPAC-UFC) e Faculdade de Economia, Administração, Atuária e Contabilidade (FEAAC-UFC). E-mail: ana.pinho@ufc.br

Antonio Caubi Ribeiro Tupinambá

ORCID: https://orcid.org/0000-0001-7786-6687

Doutor em Psicologia Organizacional; Professor Titular da Universidade Federal do Ceará (UFC). E-mail: acrtupinamba@gmail.com

Raquel Libório Feitosa

ORCID: https://orcid.org/0000-0001-9619-8362

Doutora em Psicologia pelo Programa de Pós-Graduação em Psicologia da Universidade Federal do Ceará (UFC); Psicóloga da Universidade Federal do Ceará (UFC). E-mail: raquel.feitosa@ufc.br 